\title{
To Upvote or Downvote: Parental Supervision of Screen Time on Reddit
}

Nicole Stewart

School of Communication

Simon Fraser University

\begin{abstract}
Screen time is a controversial subject in media and technology studies. Situated within the media harm debate, binary arguments have developed in discourse about the effect screen time has on people and society. The widespread use of screen-based media is the culmination of user-friendly smartphones and tablets as well as the ubiquitous nature of screen-laden media. How parents define, implement, and manage screen time is imperative to understanding how children engage with screen-based media and the observed effect it has. To understand this discourse, I conducted a social network content analysis of conversations surrounding screen time on the user-generated platform Reddit. The analysis focused on contributors' uses of the term "screen time" and the conversations relating to the implications of screen time for children. Preliminary data suggests that groups form around clusters of information that deem screen time as having a positive, negative or neutral effect - a position that also determines a parent's decision to provide unlimited or restricted access of screens to their children. The conceptual framework for this research draws from Pinch and Bijker's (1990) social construction of technology to understand how social groups form and how these groups share meanings they attach to the artifact (in this case, screens). The group formations around screen time mimic the media harm debate, with children viewed as competent (able to use technology to create, participate and build digital literacies) or vulnerable (subjected to harmful content, physical risks, and potential delays in cognitive development). The problem with the tendency to view children's screen time as positive or negative, rather than both, is it limits management strategies on how to minimize risk and maximize benefit.
\end{abstract}

Keywords: Screen time, media harm, children, SCOT

\section{Introduction}

The objective of this paper is to examine how parental sentiments form around children's screen time. Using the social bookmarking site Reddit, I conducted a social network content analysis to examine users' posts about children's screen time. The analysis of the research is presented through Pinch and Bijker's (1990) conceptual framework. Examining parental sentiments about children's 
screen time is important because screens have permeated the social structures of everyday life. Screen-based media, such as smartphones, tablets, computers, and televisions are nearly impossible to escape in today's society. Some devices, like the mobile phone, have evolved from a simple communication tool into an integral technological artifact that rarely detaches from the user. The pervasiveness of screens has not reduced the controversy over the perceived effect that screens have on the human condition. Uncovering how groups and sentiments are formed around children's screen time provides valuable information about the problems, solutions and dominant ideologies surrounding the topic.

Within this paper, I analyze forum posts on Reddit about children's screen using Pinch and Bijker's (1990) conceptual framework, which includes interpretive flexibility, social groups, problems and solutions, and a discussion of the broader context of the issue. I argue that screen time is a slippery, flexible thing that changes with time. Current groups form around ideological clusters that view children's screen time as positive, negative, neutral or mixed. The problems and solutions that emerge from these groups mirror the media harm debate, thus making screen time a debate within a debate. Parents currently deal with children's screen time however it suits the milieu best. The inherent problem with this is that social groups largely view children's screen time as positive or negative, without recognizing that screen time is positive and negative, which means parental management strategies largely ignore how to deal with the full impact that screen time has on children.

\section{Social network content analysis on Reddit}

Reddit is a bulletin board-style forum where users post, comment and vote on a variety of topics like law, news, and parenting issues like screen time. Posts are generated and ranked by users using positive and negative votes, referred to as an upvote or downvote (Haralabopoulos, Anagnostopoulos, \& Zeadally, 2015). Posts that receive a high rank are positioned on the front page of Reddit (Haralabopoulos et. al, 2015). Reddit follows a similar setup and user interaction scheme to other userpowered social news sites like Slashdot (Weninger, 2014). "Web users may access these sites anonymously (without an account) in read-only mode where they can browse postings and comments, but not contribute, vote or comment" (Weninger, 2014). To set up an account, users need a username, password and to pass a challenge-response test (e.g., Captcha-test). As a result, contributors are able to remain anonymous (Weninger, 2014). Each post typically contains a title, content and comment section (Haralabopoulos et. al, 2015). "The comment section is hosted within Reddit domain while content is usually hosted at an external domain [like YouTube] and rarely in Reddit" (Haralabopoulos et. al, 2015).

As of December 2015, Reddit had 234 million unique visitors and 8 billion page views (Reddit, 2016). The average visitor spends 11:11 minutes on the site per visit (Reddit, 2016). Fifty-three percent of visitors are male and forty-seven percent are female (Reddit, 2016). Fifty-four percent of users are based in the United States and forty-six percent of users are designated as "international" (Reddit, 2016). A report by the Pew Research Center found that 6 percent of online adults are Reddit users, there are more male users on Reddit than female users, and men between the ages of 18-29 are the most active users (Duggan and Smith, 2013). 
Reddit provides a useful framework to analyze children's screen time because: 1) the user community is active; 2) the platform is increasing in popularity; 3) the posts, comments and aggregate user data is publicly accessible; 4) the platform is rich in content related to the topic; and, 5) the users exhibit informed - yet diverse - opinions.

\section{The Current Research}

A social network content analysis was used to retrieve forum threads about children's screen time from Reddit. The purpose of the research was to uncover user perceptions about children's engagement with "screen time." On Reddit, the forum posts were collected using an internal search for "screen time children".

The research examines the discourse surrounding the controversy of screen time, particularly with respect to children, and outlines issues that parents view as potential problems and solutions. How parents define, implement, and manage screen time is imperative to understanding how children engage with screen-based media and the observed effect it has.

I examined five forum threads, totaling 224 comments. The threads were selected because they were the first forums to appear because they had the greatest amount of engagement. A PDF of each web forum was created and uploaded into NVivo 10 for analysis.

Within NVivo, the data was coded for artifact type, effect, and parental management strategies for dealing with children's screen time. The nodes that were coded for artifact type included smartphones, tablets, television, and computers (personal computers and laptops). Social groups were situated around the perceived effect or impact of children's screen time. This was coded in emotional terms, as positive, negative, neutral or mixed (positive and negative).

An example of a post that was coded as positive includes:

I... was raised on TV since basically birth, my mom loves movies and such, and coupled with it still being at least the 90s, what really inspired me were the documentaries about basic things like lion prides or how moss and fungus grows that made me get outside and get immersed.

By comparison, an example of a post that was coded as negative includes:

It's really sad when I see people taking pictures at museums or events of historic sites. Just take a while to breathe it all in first, and then take some pictures if you need to. I feel like this kind of thing is ruining our ability to remember things.

Several research limitations exist. Only a small fraction of the forums related to children's screen time were analyzed for this research. The comments on social network forums are not a representative sample. The dataset necessarily excludes lurkers, as only active participants can be analyzed. There are people who don't use social media or forums so the sample is necessarily limited to the users of Reddit. It is unknown if the comments on the analyzed forums adequately represent all sentiments and social groups that exist about children's screen time. 


\section{Applying Pinch and Bijker's framework}

What follows is an analysis of the research under Bijker and Pinch's (1990) multidirectional conceptual framework that consists of four components: interpretive flexibility, social groups, closure and stabilization, and the wider context.

\subsection{The interpretive flexibility of screen time}

The first stage in the conceptual framework is interpretive flexibility - the ability to show how technological artifacts are "culturally constructed and interpreted" (Bijker et al., 1990, p. 40). The concept of flexibility can be represented in how people interpret the artifact as well as in the object's design (Bijker et al., 1990). On Reddit, there is flexibility over the interpretation of "screen time," which mirrors the historical evolution of the term. Historically, the term "screen time" is rooted in how long (or how much time) an actor or subject appears on screen. On Reddit, it is common to find posts with high user engagement about a particular television show, movie, or character. For example, a post titled, "What is the most useless character that actually get a fair bit of screen time?" [sic] received 253 comments. The second way "screen time" is interpreted relates to the technical capacity of the screen itself. These posts examine the battery life of particular brands and models in relation to the user's screen time on the device. The third way that "screen time" is interpreted on Reddit reflects the time that people spend in front of screen-based media. The interpretive flexibility of these definitions is not permanent. Actor time on screen remained the dominant interpretation for "screen time" until screen-based media became ubiquitous in everyday life. Now, the "always-on" and "always connected" nature of screen-based devices has shifted the dominant interpretation of "screen time" to the amount of time people spend in front of screens. This new dominant interpretation of "screen time" is the basis of the following research and analysis.

Screen time is a broad term meant to include time spent in front of all screen-based media. The screen artifacts were coded to better understand the devices that users were most concerned or interested in conversing about. Forty percent of users wrote about smartphones, twenty-three percent of users wrote about tablets, twenty-one percent of users wrote about television, and sixteen percent of wrote about computers (desktop and laptop computers were coded as one unit).

\subsection{Relevant social groups form around the affective dimensions of the debate}

The second stage in the conceptual framework is relevant social groups. A key finding that emerged from the data was the idea that social groups formed along similar ideological lines. The formation of social groups is culturally constructed through ideology. The social groups that form around children's screen time have similar opinions and beliefs (ideologies). Bijker and Pinch's (1990, p. 30) note that social groups should share "the same set of meanings, attached to an artifact." Thus, the group members share the same set of meanings about children's screen time as other people who also belong to the same social group.

According to Zyga (2008), people's opinions "both influence and are influenced by our surroundings." The development of opinions is "strongly influenced" by the people a person interacts with - 
either by how strongly a person is influenced by another individual or the number of overall connections an individual has (Zyga, 2008). The opinions that people have about screen time are largely influenced by what other people in an individual's network think about screen time. According to Siebold (2014), people associate with people who are similar to them. "People with high-level formal education like to associate with the academic elite... rich people like to associate with others who are rich" and so forth (Siebold, 2014). Whether people form the sentiment that screen time is negative or positive (or whether they even think about the subject) has a lot to do with the people they are influenced by and connect with on a regular basis.

Opinions form around social groups that share the same set of meanings about children's screen time. Several groups may exist but the one thing each group has in common is that it shares the same set of values and beliefs as other people within the group. Every social group is susceptible to being influenced by media, academics and the people they engage with on a regular basis.

More than one social group can form around a thing like screen time. In the case of children's screen time, several groups appear to form, primarily around the affective dimensions of the argument. The arguments that parents make about children's screen time appear to stem from news media, which is largely modified from academic research and articles. While free from scholarly jargon, the groups form along similar lines to the ideas attributed to the various traditions within the philosophy of technology.

Bergey and Kaplan (2010) argue that "all nominal groupings are themselves cultural constructions: social schemas and that emerged through social interaction in particular contexts to fulfill conceptual and practical functions in ritualized social life." Social groups have "messy" boundaries that are constantly evolving (Bergey et al., 2010). "As a cultural phenomenon, nominal groupings should be themselves a topic for study" (Bergey et al., 2010). While nominal groupings do not have an ontological presence they are important to the social and political landscape of society (Bergey et al., 2010). The cultural construction of groupings or experiences shared by a group can "result in cultural processes" (Bergey et al., 2010).

The social groups that formed around children's screen time were ideologically based (that is, based on beliefs and opinions), which tended to have an affective element. As such, the perceived effect of children's screen time was coded as positive, negative, neutral (neither positive nor negative) or mixed (both positive and negative), as shown in Table 1. On Reddit, social groups formed around this affective nature of the argument. These social groups are groups because they share the same ideology. A space with shared ideology creates a sense of belonging. Seth Godin $(2008$, p. 3) argues: "the most powerful of our survival mechanisms is to be part of a tribe to contribute to (and take from) a group of like-minded people." One of the key observations that emerged from the research is that each group had members that supported other members who had similar beliefs and tried to denounce groups within other ideological groups. The groups were like-minded and supportive of other people with similar ideas and ideals. Godin (2008, p. 17) argues that the term "partisan" may be a criticism when hurled at a politician "but all tribes are made up of partisans, the more partisan the better."

Six percent of users believed screen time to have a mixed (both positive and negative) effect citing similar benefits and drawbacks to the negative and positive social groups. 
Twenty-seven percent of users perceived the use of screen time to be neutral (neither positive or negative in nature). One Reddit user, self-described as a professor at a university in Australia, perceived "screen time" to be neutral, noting that the evolving nature of communication means there are inevitably benefits and drawbacks: "Smartphones are great tools, but each year in-class mobile use increases and attention goes down. I think mobiles are to blame, but am I remembering simpler times [and] out of touch to the new reality?... In my mind evolution is not negative or positive, it just is. For instance, the way language changes over time might disappoint some, but there is little point complaining."

\section{Table 1}

Reddit Sentiment Groups

\begin{tabular}{cc}
\hline Sentiment Group (Tone) & Reddit (\%) \\
\hline Positive & 30 \\
Negative & 29 \\
Neutral & 27 \\
Mixed & 6 \\
Undecided/Unclear & 8 \\
\hline
\end{tabular}

Twenty-nine percent of users perceived screen time to have a negative effect. The users who thought screen time was negative formed clusters around thematic issues like the potential drawbacks, side effects and lost human skills. One user posted: "Attention [and] focus are the causalities with these little dopamine dispensing distraction machines. Yes, they are amazing tools, but how much are we really giving up?"

Thirty percent of users viewed screen time as having positive benefits for adults and children. The social group formed clusters around the benefits of screen time, most frequently citing cultural literacy, digital literacy, and keeping in touch with friends and families. One user lamented the incredible benefits of the smartphone and noted that with respect to issues like technological addiction: "It's the user that's the problem, not the phone."

\subsection{The problems and solutions that emerge from "effects" social groups}

The third component of the conceptual framework is problems and solutions (also called closure and stabilization). Within this stage, each social group plays a primary role in defining the problems; after all, a problem can only exist if "there is a social group for which it constitutes a 'problem"' (Bijker et al., 1990, p. 30). The fourth - and final - stage of the conceptual framework is examining the wider context as it pertains to screen time. The task here is "to relate the content of a technological artifact to the wider sociopolitical milieu" (Bijker et al., 1990, p. 46).

"In deciding which social groups are relevant, we must first ask whether the artifact has any meaning at all for the members of the social group under investigation" (Bijker et al., 2009, p. 30). 
The social groups that have developed around screen time on Reddit each share similar interpretations, problems and solutions around screen time - a debate that mirrors the media harm debate in children's studies. The key problems, such as how to categorize children (as vulnerable or competent) and the tradeoff between new and lost skills leads into the third component of the conceptual framework: problems and solutions (also called closure and stabilization). Within this stage, each social group plays a primary role in defining the problems; after all, a problem can only exist if "there is a social group for which it constitutes a 'problem'” (Bijker et al., 2009, p. 30). Discussions on Reddit make it possible to link the social groups with the binary arguments presented in the media harm debate.

We know that the more children go online the more risks they inadvertently or knowingly encounter (Olaffson, 2014, p. 6). The media harm debate has two sides: one that is concerned about the risk of harm to children (through media representations or appropriations) and the latter concerned about children's right to expression, exploration, and risk-taking (Drotner et al., 2008, p. 3). These binary arguments are framed through discourse as the "vulnerable child" and the "competent child" (Drotner et al., 2008, p.10). A partial reason for the academic divide is because terms like "harm" and "vulnerability" are rarely defined (Livingstone, 2007, p. 5). Media effect scholars perceive children as "vulnerable to media influence" (Livingstone, 2007, p. 5). Most research on media harm has been focused on television with limited research on new forms of media with a host of different conditions like regulation, mediation, and literacy (Livingstone, 2007, p. 15-16). Adults are in a position of power over children for reasons of care but are also "dependent upon children to secure the continuation of life" (Drotner et al., 2008, p.10). "From the point of view of children's rights, it is crucial to recognize and acknowledge that media culture is a part of children's daily lives from the earliest age" (Olafsson et al., 2014, p. 25). In 2010, a large-scale study in Finland examined a sample of 743 families with children aged 0-8 years and found that media use begins at a very early age, with the majority of 0-2 year olds listening to books, radio, and sound recordings (Olafsson et al., 2014, p. 24-25).

The dichotomy between the competent child and the vulnerable child is represented within the discussions between the social groups that perceive screen time to be negative or positive. One parent posted:

The more something is restricted, the more kids will want it... Particularly if the kid goes to school outside the home [or] has friends. They will find out what screens are, and be upset they don't have as much time as their friends. Making it available and giving them the responsibility of deciding how much they watch will make them far more likely to restrict themselves because you are trusting them, and giving them freedom...

Another parent suggested that it is important for children to learn how to navigate various platforms, including laptops and tablets, to help prepare children for the skills and competencies they will require when they go to school. "I think the difference is using technology as tools and not solely for games."

The American Academy of Pediatrics (2014) warns that "[t]elevision and other entertainment media should be avoided for infants and children under age 2 . A child's brain develops rapidly during these first years, and young children learn best by interacting with people, not screens." The potential 
for negative impacts of screen time on brain development at this age is more possible because "neural growth occurs at a rate of 700 new synapses per second" (Ernest, 2014, p. 185). Many Reddit parents avoided screen time until age 2 and continued to limit screen time until the age of 4 . One such parent said, "there's almost no benefit to offering more exposure to technology to small children and there are some risks that look like they have substantial evidence behind them." However, these parents are a minority. The research indicates that 60 percent of "parents allow their children, ages 6 to 23 months, to be exposed to some TV or video media each day" and "39 percent of families with babies, infants and preschoolers up to 4 years old" had the television on most of the time. Rachel Barr, a psychologist at Georgetown University notes that from a learning standpoint, children are slower to imitate what they see on screen than what they see in person (Guersney, 2007, p. 60). In fact, an hour of television viewing per day by 0-2 year olds was linked to a 9 percent reduction in creative play (and an 11 percent reduction on the weekend) (Guersney, 2007, p. 21).

Children model the online and offline activities of parents and siblings and primarily use technology to play games and listen to music (Livingstone et al., 2014, pp. 24-25). "Children and young people use mobile phones at increasingly young ages. Haptic and tactile interfaces of iPads and tablet devices allow very young children to relatively easily use devices" (Goggin, 2013, p. 24). "Because young people's lives are typically heavily regulated by their parents, families, communities, and institutions (such as school), and previous technologies were not portable (the telephone) or communicative (the television), mobile phones offered new possibilities for the reconfiguration of relationships with their intimates, friendship groups, peers, and families (Donald, Anderson, \& Spry, 2010)" (Goggin, 2013, p. 84).

Throughout the history of communication, new technological tools cause new skills to develop while others disappear. The discourse surrounding screen time represents optimism for the future on the part of the positive effects social group and nostalgia for the past by the negative effects social group. One user posted:

I am very concerned about many people around me being absorbed by their smartphone while walking and even driving. That's very dangerous for them, but unfortunately also for other people... There does not seem to be a world without smartphones for more and more people. Life seems to run away from them without being noticed and they do not consciously miss anything. Many of these people are like zombies.

The nostalgia for simpler times is represented by the negative effects social group, where the most coded concern was the perceived impact that screens have on face-to-face communication and "real" relationships. Similar to the user who posted about the "dopamine dispensing distraction machines" many users pose questions about the kinds of socialization skills that are being lost in a screen-centric society. One user lamented that providing children with electronic devices at the dinner table is harmful to their social development. Another user noted that "more people are going to have problems with having conversations because they aren't encouraged to at a young age. They will have been given a device to keep them quiet."

In a rebuttal to the claim of lost social skills as a result of electronics at the dinner table or restaurant, one user said: 
I do not think that the art of the civilized conversation has been cultivated well in most families, even before the advent of distracting electronic devices. However, now people are learning how to send tweets and text messages, as a substitute for a civilized conversation.

The strain that smartphones place on the parent-child relationship were the most common anecdote told about the impact of distracting devices within the household. For example, one user posted:

Just last night I was watching a movie with my son. I took out my phone just for a few minutes to look something up, and he said, "Mom, I want you to watch this with me, not be on your phone." Even though it was only a few minutes, it was enough to take away from our time together. I apologized and put the phone away, but if he hadn't said anything I might have been on it for quite a while.

The positive effects social group does not view screen time as an impediment to face-to-face communication or "real" relationships; but rather, screen time acts as an additional tool to stay connected to information and people. Parents acknowledged the benefit of screen-based technologies as a tool to connect with family at a distance: "I allow my son to Skype and Facetime with my family quite often (5 times a week around $30 \mathrm{~min}$ [ute]s each)."

Several parents tried to debunk criticisms that screen time was crippling social skills. One parent, who describes her 17-year-old son, raised with few screen time restrictions, as sensitive and compassionate said, "I do not support the correlation between screen time and decreased emotional sensitivity. I think there are more impactful factors at work there such as parent's [sic] refusal to let kids experience a full range of emotions under the pretense of 'protecting' them."

Another parent concurred:

We don't put any limits on screen time, never have. If the kid was in danger of becoming a zombie, we would, but otherwise, we figure it's better to let her figure out how to self-regulate. On school nights, she spends maybe 30 minutes in front of a screen of some sort, and on weekends, a few hours. As for empathy... and reading emotions, that's something my daughter is very adept at. She's also a pretty self-aware kid.

Even families who adopted the American Academy of Pediatrics' recommendation not to allow screen time before the age of two cited the benefits of screen-based media after this initial "cold" period. One parent emphasized that in addition to reading physical books and playing outdoors, screens, like television, are part of the child's media diet; however, when the child interacts with screens, the adults in the household engage in the same activity with the children. "Last week, we were talking about nebulae, so we put on a program about that. Basically, we use television as a tool and family bonding experience."

Another significant concern raised by the negative effects social group is the impact screen time has on emotional and physical health -with most users citing decreased social functions and obesity 
as the two health concerns impacted the most. According to the research, "excessive screen time has been linked to childhood obesity, sleep disturbances, poor school performance, hindered peer relationships, and the undermining of learning" (Ernest et al., 2014, p. 184). The more children are exposed to media violence the greater their propensity toward aggressive attitudes and the more desensitized they become to violence (Ernest et al., 2014, p. 184). In respect to the Internet, "despite the lack of evidence (and the lack of research) on harm, there is a growing body of national and international research on children's distress when they accidentally come across online pornography and other unwelcome content" (Livingstone, 2006, p. 7).

One user described mobile phones as a "morally indefensible form of technology" that, in addition to making people antisocial, l can cause "serious problems with some people's health."

Another user posted:

While the actual number is arguable, there's no question that many kids have WAY too much screen time. There are studies that show that it actually can damage your brain. There are other studies that show that it can lead to obesity and decreased social functioning.

Despite research that children have become sedentary in the age of technology and that obesity is linked to extended screen time, a study by Livingstone et al. $(2014$, p. 3) discovered that young children often lead active lives that consist of a variety of activities including sports. But mental health is also a concern, particularly for young children. Several parents cited tempter tantrums with preschool-aged children when it was time to transition away from screen-based media. One parent noted: "We've noticed tantrums correlating with her TV time, sometimes directly caused by it (as in, "your show's over now come to dinner"). When this happens we go back to zero TV for a while and carefully reintroduce."

The positive effects social group most frequently cited cultural and digital literacy as the main benefits of screen time for children. One user pointed out that he/she learned English by watching television and playing video games. "[0]nline games teach you valuable team working skills and games like Minecraft let you build amazing things and use your [brain]."

A parent who described him/herself as a member of the film industry said:

From my perspective, native cultural literary is extremely valuable, and video viewing is one aspect of cultural literacy, as are games (and of course books). So we have no limit on screen time, and we don't treat it any differently than any other kind of learning or play, though we do try to make sure that my son (age 1, but this has been our policy from the beginning of his life) is exposed to a variety of materials, particularly those that are age appropriate (but not shielding him from adult-oriented materials that don't contain excessive sex or violence).

One parent acknowledged wanting his/her child to be able to appreciate movies but wanting them to be a "treat" while another parent posted that they allow their 13-month-old to watch Baby Einstein DVDs and play with a LeapPad. "We don't completely disallow 'screen time' as we feel she needs to be technologically literate in our world, but we don't let it be a babysitter either. Everything in moderation." 


\subsection{The wider context of parental sentiments about children's screen time}

The fourth - and final - stage of the conceptual framework is examining the wider context as it pertains to screen time. The task here is "to relate the content of a technological artifact to the wider sociopolitical milieu" (Bijker et al., 2009, p. 46). Children between the ages of 0-8 are active citizens in the digital age but possess knowledge gaps with respect to access and use (Livingstone et al., 2014, p. 4). Children are often exposed to media without the skills to analyze content or purpose (Ernest et al., 2014, p. 185). Many children use devices and content outside of the recommended age range for the product or program (Livingstone et al., 2014, p. 4). One finding that emerged from the Livingstone et al. (2014, p. 32) study was the disconnect between children's actual media use compared to parental perception of use; for example, many children were accessing content that was not age appropriate because they knew how to bypass password protection settings. Parents are aware of the "hyperbolic claims" that technology has "educational benefits but [are] not necessarily convinced by them" (Livingstone et al., 2014, p. 26).

The largest social and political factor that emerged from the data is how various households choose to manage screen time for their children. The management strategies by parents varied from strict regulation of screen time to an unlimited amount of screen time. The most coded sentiment surrounded the idea that parents and children do not, and should not, have the same screen time rules. One user said: "[K]ids are different to adults in many ways that make them more vulnerable to media, so it makes sense to restrict their media consumption more than an adult, just like you wouldn't feed a small child an adult portion of food."

Another user implemented a strategy called "adult privilege" whereby various household members receive different privileges with respect to screen time: "We are planning on using it to negotiate why she may get opportunities that her coming younger sibling might not... Kids think fairness means the same for everyone. I think this is helping her learn fairness has context."

Having age-appropriate rules was a common management tactic for screen time use. Another user posted, "I have 4 children....I have different rules for each of them, too, based on age and personality...they are different people and need different guidance... Little brains are not fully developed...hence less screen time more books."

Most of the users cited limiting screen time to some extent. For example, some parents restricted screen time before bed so it wouldn't affect sleep or on school nights when homework needs to be done. Some families allowed screen time on school nights after homework was done or to assist with homework. Other users agreed that there should be some control placed on screen time for children but weren't sure what those limitations should be.

Some users did not agree with placing any limitations on screen time, stating, "I personally think the whole 'no screen time' thing is absurd. We live in a technological society. It's better to teach moderation and appropriate use with something like smartphones rather than banning them completely. It will just result in your children sneaking around behind your back to do things if they aren't allowed it at all when at home."

Other parents acknowledged that screen time was used as a way to give parents a break. One user noted that in an effort to sleep in on weekends, her children were permitted to watch cartoons 
for a couple of hours in the morning. Another parent noted that electronic devices are a must at restaurants so they can "rest, eat a quick meal, and try to relax a bit away from our toy- and diaperstrewn house."

A key problem with the parental management strategies that surfaced within the discussions is that because many parents side with the idea that children's screen time is positive or negative, each management strategy largely ignores the other element of the debate. Children's screen time is not positive or negative; it is both, and it is imperative for parents to acknowledge that fact when managing screen time to help minimize risk and maximize the benefits of time in front of screens.

\section{Conclusion}

Screen time is a flexible subject with a slippery, ever-changing definition. Parental sentiments about children's screen time on Reddit develop in social groups formed around the affective dimensions of the debate - positive, negative, neutral or mixed. The "effect" groups mirror the media harm debate, which positions children as either vulnerable or competent with respect to their media and screen usage. Parental management strategies about children's screen time differ and each household currently does what is best for the family unit. The key problem that emerges is that parents create management strategies based on their belief that screen time is positive or negative, rather than both, which means children do not always receive supervision in a way that minimizes risk and maximizes the potential of the artifact.

\section{References}

American Academy of Pediatrics (2014), Retrieved from:

https://www.aap.org/en-us/advocacy-and-policy/aap-health-initiatives/pages/media-and-children.aspx

Bergey, B.W., \& Kaplan, A. (2010). What do social groups have to do with culture? The crucial role of shared experience. Frontiers in Psychology, 1, 199. doi: 10.3389/fpsyg.2010.00199

Bijker, W. E., Hughes, T. P., \& Pinch, T. J. (. J. ). (2009). The Social construction of facts and artifacts: Or how the sociology of science and the sociology of technology might benefit each other. Retrieved from: http://www.sfu.ca/ andrewf/

Bijker, W. E., Hughes, T. P., \& Pinch, T. J. (. J. ). (2012). The social construction of technological systems: New directions in the sociology and history of technology (anniv. ed.). Cambridge, Mass: MIT Press.

Drotner, K., \& Livingstone, S. (2008) Volume introduction (and introductions to parts I-IV). In K. Drotner \& S. Livingstone (eds.), The international handbook of children, media and culture. London, UK: Sage Publishing.

Duggan, M. \& Smith, A. (2013). 6\% of online adults are Reddit users. PEW Research Center. Retrieved from: http://www.pewinternet.org/files/old-media/Files/Reports/2013/PIP_reddit_usage_2013.pdf 
Ernest, J., Causey, C., Newton, A., Sharkins, K., Summerlin, J., \& Albaiz, N. (2014). Extending the global dialogue about media, technology, screen time, and young children. Childhood Education, 90:3, 182-191. doi: 10.1080/00094056.2014.910046

Godin, S. (2008). Tribes: We need you to lead us. New York: Penguin Group.

Goggin, G. (2013). Youth culture and mobiles. Mobile Media \& Communication, 1(1), 83-88. doi:10.1177/2050157912464489

Guernsey, L. (2007). Into the minds of babes: How screen time affects children from birth to age five. New York: Basic Books.

Haralabopoulos, G., Anagnostopoulos, I., \& Zeadally, S. (2015). Lifespan and propagation of information in online social networks: A case study based on Reddit. Journal of Network and Computer Applications, 56, 88-100.

Lim, S. S. (2013). On mobile communication and youth "deviance": Beyond moral, media and mobile panics. Mobile Media \& Communication, 1(1), 96-101. doi:10.1177/2050157912459503

Livingstone, S. (2008) Engaging with media - a matter of literacy? Communication, culture \& critique, 1 (1). pp. 51-62. doi: 10.1111/j.1753-9137.2007.00006.x

Livingstone, Sonia (2007) Do the media harm children?: reflections on new

approaches to an old problem. Journal of children and media, 1 (1). pp. 5-14. doi: 10.1080/17482790601005009

Livingstone, S., Marsh, J., Plowman, L., Ottovordemgentschenfelde, S., \& Fletcher-Watson, Ben (2014) Young children (0-8) and digital technology: a qualitative exploratory study - national report - UK. Joint Research Centre, European Commission, Luxembourg.

Ólafsson, K., Livingstone, S., \& Haddon, L. (2014). Children's use of online technologies in Europe: A review of the European evidence base (Rev. ed.). LSE, London: EU Kids Online.

Reddit (2016). Retrieved from: https://about.reddit.com/

Siebold, S. (2014). How rich people choose their friends. Business Insider. Retrieved from: http://www.businessinsider.com/rich-people-choose-friends-2014-12

Zyga, L. (2008). Physicists model how we form opinions. Phys.org. Retrieved from: http://phys.org/news/2008-04-physicists-opinions.html 